\title{
Seroprevalence of Rubella IgG in Pregnant Females
}

\author{
Huda Rana', Rijja Chaudhary ${ }^{2}$, Saeed Alam ${ }^{3}$, Wajiha Mahjabeen \\ 1,2 Student MBBS, 2 Professor, Department of Pathology, ${ }^{3}$ Associate Professor, Department of Pathology \\ Islamabad Medical and Dental College, Islamabad
}

\begin{abstract}
Objective: To find out the seropositivity of IgG rubella in pregnant women in Pakistan, to assess the prevalence of infection and to estimate the requirement of vaccination.

Patients and Methods: In this retrospective cross sectional study total 730 females who visited Salma Kafeel Fertility Clinic (Rawalpindi) for antenatal checkup were inducted. Their age range was 18-45 years. The study was carried out from 2011-2016. ELISA serological testing was used to check the seroprevalence of Rubella IgG.

Results: In total the 730 subjects were included in the study and divided in four groups according to their ages. The results were as follows: group 1(18-20yrs) 100\% positive, group 2 (21-30yrs) 90.5\% positive, group 3 (31-40yrs) 93.5\% positive and lastly group 4 (>40yrs) 95\%positive. Overall $92.32 \%$ seropositivity was seen while $7.70 \%$ were found at risk of developing infection.

Conclusion: There is a high prevalence of Rubella in females of child bearing age in Pakistan. This is alarming as it can result in high risk of CRS in newborns and fetal death. Therefore, women planning to become pregnant, who do not have natural immunity to rubella should be vaccinated at least 4 weeks prior to conception.

Key words: IgG, Rubella, Seropositivity

Author's Contribution $\quad$ Address of Correspondence

${ }^{1}$ Conception, synthesis, planning of research and manuscript writing Interpretation and discussion

${ }^{2-4}$ Data analysis, interpretation and manuscript writing, Active participation in data collection.
\end{abstract}

Cite this article. Rana H, Chaudary R, Alam S, Mahjabeen W. Seroprevalence of Rubella lgG in Pregnant Women in Pakistan. JIMDC.2018; 7(4):295-298

\section{Introduction}

Rubella (German measles) is an acute, contagious viral infection. It is a generally mild condition in children, but may have serious consequences in pregnant women causing fetal deaths, or congenital defects known as Congenital Rubella Syndrome (CRS). The most common birth defects from CRS can include deafness, cataracts, heart problems, mental retardation, and cerebral palsy. Late onset manifestations of CRS include glaucoma, thyroiditis, and Diabetes Mellitus. ${ }^{1}$ In 1981-1983, the incidence of CRS in America was 0.9-1/1000. ${ }^{2}$ However, since 2012, only six babies with CRS have been reported. ${ }^{3}$ Infection with Rubella is most severe when the mother is infected during the first trimester of pregnancy. According to a study conducted in $1987,72 \%$ of the married women, $77 \%$ of the pregnant females, and $51.75 \%$ of the pre-pubertal females were immune to Rubella in Pakistan. ${ }^{4,5}$

Rubella infections can be easily prevented if the adult women of child bearing age are vaccinated before they become pregnant. According to the Centers for Disease Control and Prevention, before the introduction of the measles vaccine in 1963, 4 babies in every 1000 live 
births were born with CRS. Large scale vaccinations over the past decade have almost eliminated Rubella and CRS in the developed countries as well as in many developing countries. WHO Region of the America became the first in the world to be declared free of endemic transmission of Rubella in $2015 .^{6}$

In Pakistan Rubella vaccination is not the part of national expanded program of immunization (EPI). Data regarding prevalence of Rubella antibodies among females of reproductive age is also insufficient. The current study is planned in order to find out seroprevalence of rubella antibodies and to high light the importance of vaccination in this population.

\section{Patients and Methods}

The retrospective cross sectional study was carried out at Salma \& Kafeel fertility clinic, Islamabad. Sample size was calculated by using open epi calculator, and parameters were based on $83.4 \%$ seroprevalence found in reproductive age with $99.9 \%$ confidence levels. ${ }^{7}$ Calculated sample size was 600 individuals. It was a single center study that's why we inducted 730 subjects to further strengthen our data statistically through non probability convenient sampling. Data of total 730 pregnant females who visited the clinic from year 20112016 for antenatal checkup was analyzed. Enzyme linked immunosorbent assay (ELISA) was performed to determine the presence of rubella specific immunoglobulin $G(\lg G)$. Data was entered as number and percentage. Chi square test was applied to determine the association of Rubella infection with age.

\section{Results}

Total 730 subjects with age range $15-45$ years were divided in four groups according to their ages; group 1 (17-20 years), group 2 (21-30 years), group 3 (31-40 years) and group 4 (40 years \& above). Group 1 showed $100 \%$ positivity while in group 2, 3 and 4 there were $90.5 \%, 93.5 \%$ and $95 \%$ patients positive for Rubella IgG, respectively. Rubella IgG positivity shows that the women already had gained immunity to the infection, either because of previous infection or due to vaccination. The overall seropositivity was $92.3 \%$, indicated the presence of immunity for Rubella infection and the remaining $7.70 \%$ females were at risk for developing Rubella infection.

Table 1: Association of rubella infection with age
(n=730)

\section{Discussion}

Rubella, also known as German measles, is a contagious viral disease. It is a highly infectious disease, spread via air through coughs of infected people. In non-pregnant women, it causes relatively mild symptoms such as coryza, low grade fever, mild redness of the eye, and an associated characteristic exanthematous rash. Infections in the pregnant women especially during the first trimester of pregnancy can be transmitted to the fetus, causing Congenital Rubella Syndrome. Congenital Rubella Syndrome is a major cause of several preventable life threatening disabilities. Ophthalmic defects, mental retardation, heart anomalies, sensorineural deafness are some common manifestations. ${ }^{8}$ There is insufficient data about the current immune status of pregnant women against Rubella in our country. We carried out a study to assess the seroprevalence of Rubella lgG in pregnant females. IgG Serology testing can be done to diagnose immunity to Rubella. ${ }^{9}$ Rubella virus infection confers lifelong immunity but it may also be achieved through vaccination. Therefore, this study also helps us to estimate the need for Rubella vaccination among women in Pakistan as the MMR vaccine is not yet a part of the national EPI program. Although it is ideal that every woman of child bearing age should also be tested for Rubella infection and be vaccinated accordingly, at the very least these measures should be carried out in women referring to an infertility clinic. We collected data from 730 women seeking antenatal treatment at Salma and Kafeel Medical center and Infertility Services in Islamabad, Pakistan. Our results cannot represent the immune status of the entire female population of Pakistan, but it serves as a template for similar studies to be carried out in the more rural areas. The seroprevalence of Rubella antibodies among our study population was 
positive for $92.32 \%$ with the remaining $7.6 \%$ testing negative and therefore susceptible to Rubella. The highest degree of susceptibility was among women of ages $21-30$ years with a seronegativity of $4.52 \%$, then susceptibility decreases with age. This means that in the best reproductive age (20-30 years ) considerable number of women if start reproduction without being immunized prior to conception can have a chance of getting CRS. A Pakistani study carried out in 2006 estimated 3 in 100 infants as confirmed cases of Rubella. ${ }^{10}$

Our results are quite similar to a study conducted at Multan, Pakistan in females of reproductive age. Their study revealed $93.33 \%$ seropositivity while $5 \%$ females were seronegative and $3 \%$ were in intermediate range. ${ }^{11}$ Our results were also in concordant to a study conducted in 2016 at Kerala, India where $94.3 \%$ females were lgG positive. The mean age of the subjects $(n=70)$ was $24.7 \pm 4.3$ years and data collection was done by ELISA. ${ }^{12}$ However, a cross sectional study carried out between 2012-2014 in the district of Bijapur, showed only $31.66 \%$ being seropositive. ${ }^{13}$ Total $88.2 \%$ of the females, in a study population of 339, in Egypt were positive for Rubella antibodies. Immunity was higher in the 25-30 age group as compared to the younger 20-25 age group. ${ }^{14}$

In Shiraz, a city in southern Iran, a study was conducted to find out the seroprevalence of Anti rubella and Anti measles $\lg \mathrm{G}$ in pregnant women. The results showed that 96\% were positive and immune to Rubella with mean geometric concentration being $14.9 \mathrm{IU} / \mathrm{mL} .{ }^{15}$ This helps us compare our country's immune status to other developing countries with an aim to collectively raise the immune status of these countries like that of the developed countries. The reported cases of Rubella in Pakistan have increased from 282 in 2015 to 648 in 2016. The reported cases in the United States was 5 in 2015 and 0 in 2016. ${ }^{16}$ This remarkable contrast is due to the efficient vaccination programs in USA. In children, the first recommended dose is given at 12 months of age and the second at 4 years. Women planning to become pregnant should be vaccinated at least 4 weeks prior to conception. ${ }^{17}$ Since the introduction of Rubella vaccine in the Canadian vaccination program in 1969, the incidence of Rubella has had a considerable decrease with the exception of some outbreaks over the years. In a retrospective observational study conducted in Alberta in 2002-2005, 91\% subjects tested positive. There was an increase in seronegativity from $8.2 \%$ in 2002 to $9.9 \%$ in $2005 .{ }^{18}$ One limitation of this study was convenience sampling from single center in main city Islamabad. That's why results may not fully represent the whole population. In future, further multi center studies with appropriate age and pregnancy duration stratification should be planned to overcome the bias.

\section{Conclusion}

The study concludes that $7.70 \%$ females of child bearing age are at risk for developing rubella infection in Pakistan. This is alarming as it can result in high risk of congenital rubella syndrome in newborns and fetal deaths. It is suggested that women planning to become pregnant and don't have natural immunity to rubella should be vaccinated at least 4 weeks prior to conception.

\section{References}

1. Robertson SE, Featherstone DA, Gacic-Dobo M, Hersh BS. Rubella and congenital rubella syndrome: global update. Revista Panamericana de salud publica. 2003;14(5):306-15.

2. Control CfD. Rubella and congenital rubella--United States, 1980-1983. MMWR Morbidity and mortality weekly report. 1983;32(39):505.

3. McLean H, Redd S, Abernathy E, Icenogle J, Wallace $G$. Congenital rubella syndrome. Manual for the surveillance of vaccine-preventable diseases. 2012.

4. Ishtiaq A. Rubella susceptibility; a study of 200 college students and hospital employees. Pharma News. 1987.

5. Azmi F, lqbal J, Rab A, Khan M, Amin A. Prevalence of anti-rubella antibodies in pregnant and prepubertal females--a preliminary study. JPMA The Journal of the Pakistan Medical Association. 1987;37(1):6.

6. Watson JC, Hadler SC, Dykewicz CA, Reef S, Phillips L. Measles, Mumps, and Rubella-Vaccine Use and Strategies for Elimination of Measles, Rubella, and Congenital Rubella Syndrome and Control of Mumps: Recommendations of the Advisory Committee on Immunization Practices (ACIP). Vol. 47/No. RR-8. Centers for disease control atlanta ga, 1998.

7. Ali S, Khan FA, Mian AA, Afzal MS. Seroprevalence of cytomegalovirus, herpes simplex virus and rubella virus among pregnant women in KPK province of 
Pakistan. The Journal of Infection in Developing Countries. 2014;8(03):389-90.

8. Imam H, Yasmin M, Ahsan CR, Nessa J. Pregnant women in and around dhaka city: are their children at risk of developing congenital rubella syndrome? Indian journal of microbiology. 2010;50(4):443-8.

9. Roush SW, Beall B, Cassiday P, Gentsch J, Icenogle J, Mayer L, Oberste SM, Payne DC, Rota P, Schmid DS, Shaw M. Laboratory support for the surveillance of vaccine-preventable diseases. VPD Surveillance. Manual. 1999.

10. Hussain N, Jaffery G, Hasnain S, Anwar K. Seroprevalence of Rubella Ig $G$ and IgM antibodies in infants suspected of having Rubella infection. Biomedica. 2006;22:25-30.

11. Quddusi H, Sultana H, Sajid KM, Sabih D, Akhtar MS. Seroprevalance of rubella antibodies among women of reproductive age: A study at Nishtar Hospital Multan. Annals of King Edward Medical University. 2016;12(4).

12. Jayakrishnan $T$, Murkoth A, George B, Rao B, Vidya K. Sero Prevalence of Rubella Immunity in Pregnant Women in Kerala, India. International Journal of Medicine and Public Health. 2016;6(4).

13. Shilpi G, Praveen R, Manpreet K. Seroprevalence of Rubella antibodies in women of reproductive age group. National J Lab Med. 2015;4:9-12.
14. Gadallah M, El Sayed N, Kandeel A, Moussa I, Mohsen A, Dewedar S. Seroprevalence of rubella antibodies among adult Egyptian females aged 20-30 years. Is there a need for rubella vaccination? Central European journal of public health. 2014;22(4): 282.

15. Honarvar B, Moghadami M, Moattari A, Emami A, Odoomi N, Lankarani KB. Seroprevalence of antirubella and anti-measles $\lg \mathrm{G}$ antibodies in pregnant women in Shiraz, Southern Iran: outcomes of a nationwide measles-rubella mass vaccination campaign. PloS one. 2013;8(1): e55043.

16. WHO. WHO vaccine-preventable diseases: monitoring system. 2017 global summary. Incidence time series for Pakistan (PAK) 2017 [updated updated 2017 Sept 6]. Available from: http://apps.who.int/immunization_monitoring/globalsu mmary/incidences?c=PAK. .

17. National Center for Immunization and Respiratory Diseases (NCIRD) DoVD. Rubella (germam measles, three day measles); 2017.

18. Kearns MJ, Plitt SS, Lee BE, Robinson JL. Rubella immunity among pregnant women in a Canadian provincial screening program. Canadian Journal of Infectious Diseases and Medical Microbiology. 2009;20(3):73-7. 